\title{
COLANGIOGRAFIA PEROPERATÓRIA SISTEMÁTICA EM COLECISTECTOMIA VIDEOLAPAROSCÓPICA
}

\section{SYSTEMATIC CHOLANGIOGRAPHY DURING LAPAROSCOPIC CHOLECYSTECTOMY}

\author{
Edson Yuzur Yasojima, TCBC-PA ${ }^{1}$ \\ Gaspar de Jesus Lopes Filho, TCBC-SP²
}

\begin{abstract}
RESUMO: Objetivo: Analisar os resultados de colangiografias peroperatórias (CPO), realizadas sistematicamente durante colecistectomias videolaparoscópicas (CVL). Método: No período de janeiro de 1998 a dezembro de 1999, foram realizadas CVL em 250 pacientes não selecionados, 65 (26\%) do sexo masculino e 185 (74\%) do sexo feminino, independente da presença ou não de sinais clínicos, laboratoriais, ultra-sonográficos ou de outros métodos diagnósticos, indicativos de coledocolitíase ou outras doenças da via biliar principal. Resultados: A CPO pôde ser realizada em 237 (94,6\%) pacientes. Foram encontradas 31 (13\%) alterações, entre as quais seis $(2,5 \%)$ casos de coledocolitíase e $25(10,5 \%)$ casos de anomalias anatômicas. Conclusão: A CPO, durante a CVL, mostrou-se um procedimento seguro, sem complicações relacionadas ao exame, definindo a anatomia, prevenindo as lesões biliares e permitindo a detecção de coledocolitíase insuspeitada.
\end{abstract}

Descritores: Colangiografia; Colecistectomia; Litíase biliar, Videolaparoscopia.

\section{INTRODUÇÃO}

A colangiografia operatória foi descrita por Pablo Mirizzi e sua utilização sistemática em colecistectomias tem sido recomendada desde 1948, assim como enfatizada a sua importância por diversos autores ${ }^{1-5}$. Seu uso rotineiro, no entanto, permanece controverso ${ }^{6-18}$.

A técnica não só fornece ao cirurgião um meio de avaliar as alterações coledocianas, como também proporciona um meio objetivo de identificar a anatomia do ducto biliar. Isto permite uma dissecção mais segura e uma colocação acurada de clipes no ducto cístico, permitindo, ainda, a imediata identificação de eventuais lesões ${ }^{3,10}$. Embora o uso sistemático da colangiografia durante a colecistectomia seja defendido por muitos autores, outros argumentam que a colangiografia, realizada de forma rotineira, não tem propiciado resultados correspondentes ao seu custo ${ }^{11}$. De maneira geral, entretanto, a maioria dos cirurgiões concorda em que a colangiografia desempenha um papel de importância considerável na abordagem cirúrgica de doenças do trato biliar. Esta é, portanto, uma técnica que todo cirurgião geral deve estar preparado para reali$\operatorname{zar}^{12}$.

Com o advento da colecistectomia videolaparoscópica, a discussão, que havia arrefecido, recrudesceu com várias ramificações, discutindo-se, inclusive, a validade de se operarem doentes portadores de litíase da via biliar principal por videolaparoscopia ${ }^{11}$.

O diagnóstico da litíase assintomática da via biliar principal e da papilite, embora importante, já não representa mais a principal indicação da necessidade de efetuar a colangiografia peroperatória ${ }^{13}$.

A preocupação com as alterações anatômicas e a segurança da dissecção do pedículo da vesícula biliar, realizada sob visão bidimensional do monitor de vídeo, sem possibilidade de palpação das estruturas, representam hoje as indicações mais freqüentes para a realização da colangiografia peroperatória ${ }^{12}$.

1. Mestre em Gastroenterologia Cirúrgica pela Universidade Federal de São Paulo - Escola Paulista de Medicina. Professor da Disciplina de Técnica Operatória, Cirurgia Experimental e Anestesia e da Disciplina de Clínica Cirúrgica I do Departamento de Saúde Integrada do Curso de Medicina da Universidade do Estado do Pará.

2. Professor Livre-docente do Departamento de Cirurgia em Gastroenterologia Cirúrgica pela Universidade Federal de São Paulo - Escola Paulista de Medicina

Recebido em 21/05/2001.

Aceito para publicação em 26/02/2002.

Trabalho realizado no Hospital Amazônia da Beneficência Nipo-Brasileira da Amazônia em Belém - Estado do Pará. 
Assim, existem cirurgiões que a realizam rotineiramen$\mathrm{te}^{5,12,14}$, outros apenas em casos selecionados ${ }^{7,13,15} \mathrm{e}$, finalmente, um grupo que nunca se utiliza deste recurso ${ }^{16,17}$.

A lesão da via biliar principal ocorre em $0,1 \%$ a $0,5 \%$ dos casos de colecistectomia convencional nos Estados Unidos. Naquele país, à semelhança da Europa, a maioria dos cirurgiões somente realiza a colangiografia peroperatória em casos selecionados ${ }^{18}$. Berci ${ }^{19}$, contrariamente, realizou-a rotineiramente e, em 4.000 colecistectomias convencionais, teve apenas quatro casos $(0,1 \%)$ de lesão ductal.

Embora já existam aparelhos de três dimensões sendo comercializados, a maioria dos cirurgiões trabalha com câmera de duas dimensões ${ }^{20}$. Na videocirurgia há, portanto, até o momento, perda ou diminuição da visão de profundidade. A sensação de profundidade é obtida através de aprendizado, comparando o tamanho das pinças com o tamanho real que foi memorizado ${ }^{14}$. Durante a videocirurgia, os cirurgiões não têm a possibilidade de visão da árvore biliar de cima para baixo, como na cirurgia convencional. A visão, dada a entrada do telescópio (óptica) pela cicatriz umbilical, será sempre tangencial, ainda que esse problema possa ser minorado com a utilização de telescópios de visão angular de $30^{\circ}$ ou $45^{\circ 14}$.

Assim, a colangiografia constitui um importante método radiológico utilizado durante a cirurgia das vias biliares, com o objetivo de definir a anatomia e diagnosticar suas possíveis variações e, ainda, o número, o tamanho e a posição de cálculos intraductais, quando presentes $^{2,16,21}$.

No sentido de acrescentar uma contribuição para o estudo desta controvérsia existente na literatura, o objetivo deste trabalho foi avaliar os resultados obtidos com a realização sistemática da colangiografia durante a colecistectomia videolaparoscópica em doentes portadores de litíase vesicular, operados eletivamente e de urgência.

\section{MÉTODO}

Foram realizadas colecistectomias videolaparoscópicas (CVL) com a utilização sistemática da colangiografia peroperatória (CPO), em pacientes de ambos os sexos, independente da presença, ou não, de sinais prévios, clínicos, laboratoriais, ultra-sonográficos ou por outros métodos diagnósticos (colangiorressonância magnética, colangiopancreatografia retrógrada endoscópica), sugestivos de coledocolitíase ou de outras doenças das vias biliares, assim como de possíveis alterações anatômicas da árvore biliar.

As colecistectomias videolaparoscópicas foram realizadas no Hospital Amazônia da Beneficência Nipo-Brasileira da Amazônia, localizado na cidade de Belém (Pará), por uma mesma equipe cirúrgica, no período de janeiro de 1998 a dezembro de 1999.

Protocolos organizados em formato de fichas foram elaborados, com dados de identificação, queixas principais, sintomas clínicos compatíveis com colecistopatia litiásica, e exames complementares pré-operatórios (incluindo ultra-sonografia e exames laboratoriais) com a prévia permissão da Instituição e dos pacientes incluídos na pesquisa.

A colangiografia peroperatória (CPO) foi proposta em 250 pacientes submetidos à colecistectomia videolaparoscópica (CVL), e foi possível em 237 pacientes (94,6\%).

Em 13 doentes $(5,4 \%)$ não foi possível a realização da CPO e a distribuição das causas de insucesso está apresentada na Tabela 1 .

Tabela 1

Causas de insucesso

\begin{tabular}{l|c|c}
\hline Causas de insucesso & $\begin{array}{c}\text { No de } \\
\text { doentes }\end{array}$ & $\%$ \\
\hline Dificuldade de cateterização do cístico & 6 & 46,1 \\
Cístico fino & 5 & 38,5 \\
Cálculo impactado no cístico & 1 & 7,7 \\
Fibrose do cístico & 1 & 7,7 \\
\hline Total & 13 & 100,0 \\
\hline
\end{tabular}

A idade dos pacientes variou de quatro a 89 anos e a faixa etária mais acometida situou-se entre 40 a 50 anos, com mediana de 45 anos.

A distribuição dos doentes em relação ao sexo revelou uma predominância do sexo feminino, na proporção de $3: 1$.

A distribuição dos doentes quanto à raça revelou uma predominância da cor parda, seguida de cor branca, amarela e negra.

Os sinais e sintomas mais encontrados foram a dor epigástrica e a dor no hipocôndrio direito.

A técnica percutânea de colangiografia foi empregada neste estudo. Isto acarretou, necessariamente, a passagem do cateter através de uma agulha, utilizando uma punção separada: um "quinto" acesso. A agulha era colocada de maneira que o cateter entrasse na cavidade abdominal pelo mesmo eixo do ducto cístico, o que facilita a passagem do cateter. Com o cateter passado através da agulha para dentro da cavidade abdominal, uma tesoura era introduzida, através do trocarte lateral ou epigástrico, e utilizada para efetuar uma pequena incisão no ducto cístico. O ducto cístico era isolado próximo à vesícula biliar, para eliminar a possibilidade de confusão com o ducto colédoco. O cateter era fixado com um clipe, tomando-se o cuidado necessário para não apertá-lo demais. Isto era conseguido injetando-se solução salina através do cateter, à medida que o clipe era fechado. A pressão cessava exatamente no momento em que se percebia resistência ao fluxo.

A seguir, os trocartes eram tracionados em direção à parede abdominal e o pneumoperitônio era esvaziado.

A colangiografia era, então, realizada de maneira usual, utilizando-se técnica de filme estático em dois tempos, com um aparelho de raios-X móvel, em uma incidência de raios que variava de 80 a 90KV em 100MA, impressos em filmes de $24 \times 30 \mathrm{~cm}$. A colangiografia de carga dupla foi a preferida neste estudo. 
A primeira injeção era efetuada, utilizando aproximadamente $5 \mathrm{ml}$ de contraste a $50 \%$, à base de iodamida meglumina (Uromiromâ), seguida de uma segunda injeção de $10 \mathrm{ml}$ de contraste com a mesma concentração.

A primeira injeção permita a visualização de pequenos cálculos que, eventualmente, estivessem presentes. A segunda injeção identificava a anatomia ductal, e qualquer deficiência no preenchimento do sistema biliar superior.

Com a colangiografia realizada e estudada pela equipe, era identificado também o clipe do cístico. Adicionalmente, o trajeto do ducto cístico era avaliado e a junção cístico-colédoco confirmada. A visão do contraste dentro do duodeno é obrigatória, assim como a visão dos ductos hepáticos direito e esquerdo. Depois de completar e revisar a colangiografia, era retirado o cateter, dois clipes eram colocados no lado proximal do cístico que era seccionado com tesoura e, em seguida, a artéria também era seccionada após ligadura. A vesícula biliar era apreendida na bolsa de Hartmann e elevada ao mesmo tempo em que era afastada inferiormente do fígado. O eletrocautério era usado para separar a vesícula biliar do leito do fígado, realizando-se meticulosamente a hemostasia.

\section{RESULTADOS}

Entre os 237 pacientes nos quais foram realizadas as colangiografias, o resultado foi considerado normal em 206 casos $(87 \%)$ e alterado em 31 casos (13\%). Dentre estes, identificou-se colédoco dilatado; coledocolitíase; ducto cístico dilatado; cístico espiralado; implantação baixa do cístico; implantação alta do cístico e cístico longo (Tabela 2).

Tabela 2

Alterações encontradas

\begin{tabular}{l|c|c}
\hline Alterações & $\begin{array}{c}\text { No de } \\
\text { doentes }\end{array}$ & $\%$ \\
\hline Colédoco dilatado & 8 & 25,8 \\
Cálculo no colédoco & 6 & 19,3 \\
Ducto cístico dilatado & 5 & 16,0 \\
Cístico espiralado & 4 & 13,0 \\
Implantação baixa do cístico & 4 & 13,0 \\
Implantação alta do cístico & 2 & 6,4 \\
Cístico longo & 2 & 100,0 \\
\hline Total & 31 & \\
\hline
\end{tabular}

Podemos dividir as alterações verificadas em dois grupos: as alterações anatômicas e as alterações patológicas.

Entre as alterações anatômicas verificamos a implantação baixa do cístico (Figura 1) em quatro casos (1,7\%); a implantação alta do cístico (Figura 2) em dois casos $(0,8 \%)$; cístico longo em dois casos $(0,8 \%)$ e cístico espiralado em quatro casos $(1,7 \%)$.

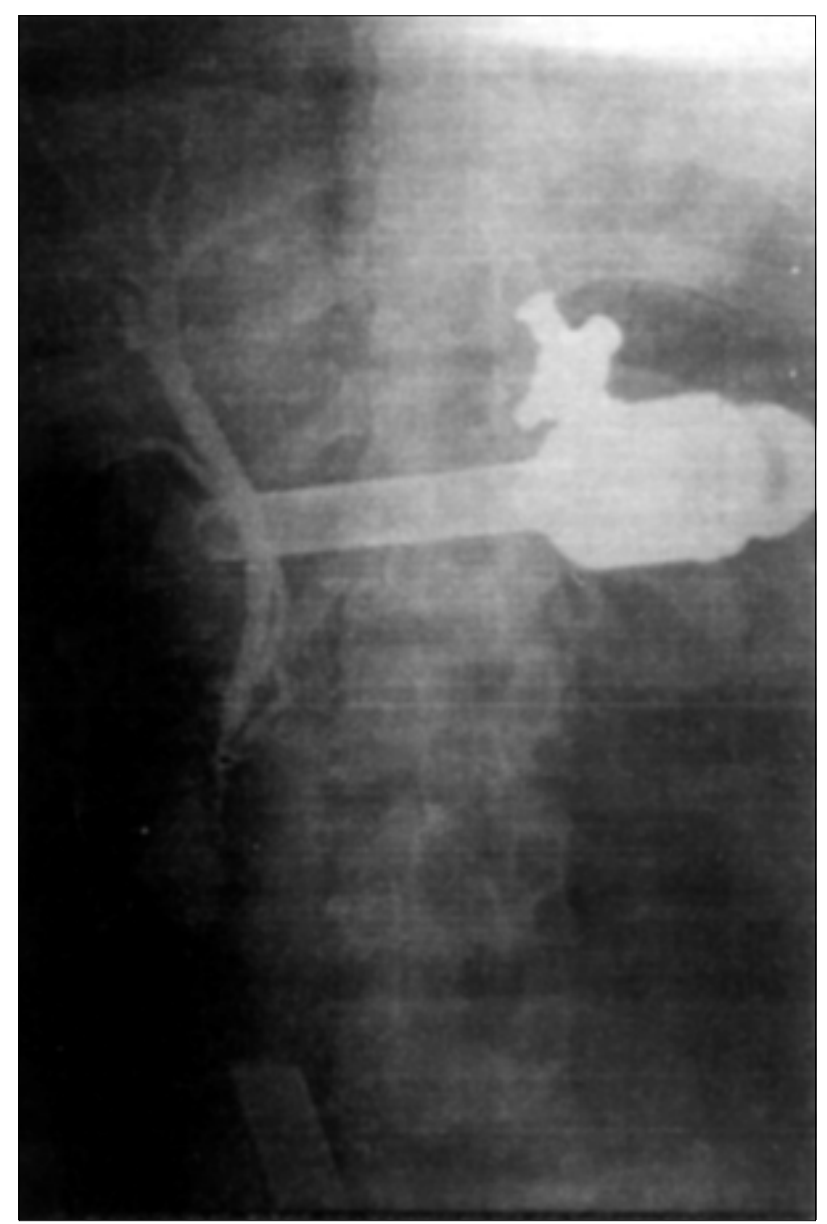

Figura 1 - Cístico longo, com implantação baixa pelo lado oposto no ducto hepático comum.

Entre as alterações patológicas, verificamos a presença de cálculos no colédoco em seis casos $(2,5 \%)$, e em três destes $(1,3 \%)$ havia coledocolitíase insuspeitada (Figuras 3 e 4$)$.

Verificamos ducto cístico dilatado em cinco casos $(2,1 \%)$ e colédoco dilatado em oito casos $(3,4 \%)$.

Dez pacientes apresentavam colecistite aguda e foi possível realizar a colangiografia peroperatória, sendo o resultado considerado normal em todos.

Não houve dificuldade de esvaziamento do contraste para o duodeno em nenhum doente desta casuística.

Dos seis casos $(2,5 \%)$ em que foram detectados cálculos na via biliar principal, em cinco a colecistectomia videolaparoscópica teve prosseguimento e foi completada, sendo a retirada do cálculo efetuada no período pósoperatório, por via endoscópica. Estes cinco casos evoluíram sem complicações no período pós-operatório precoce e tardio. No caso restante foi realizada a retirada cirúrgica dos cálculos, pelo fato de o paciente apresentar uma gastrectomia parcial com reconstrução do trânsito pela técnica de Billroth II, sem complicações no pós-operatório precoce e tardio. 


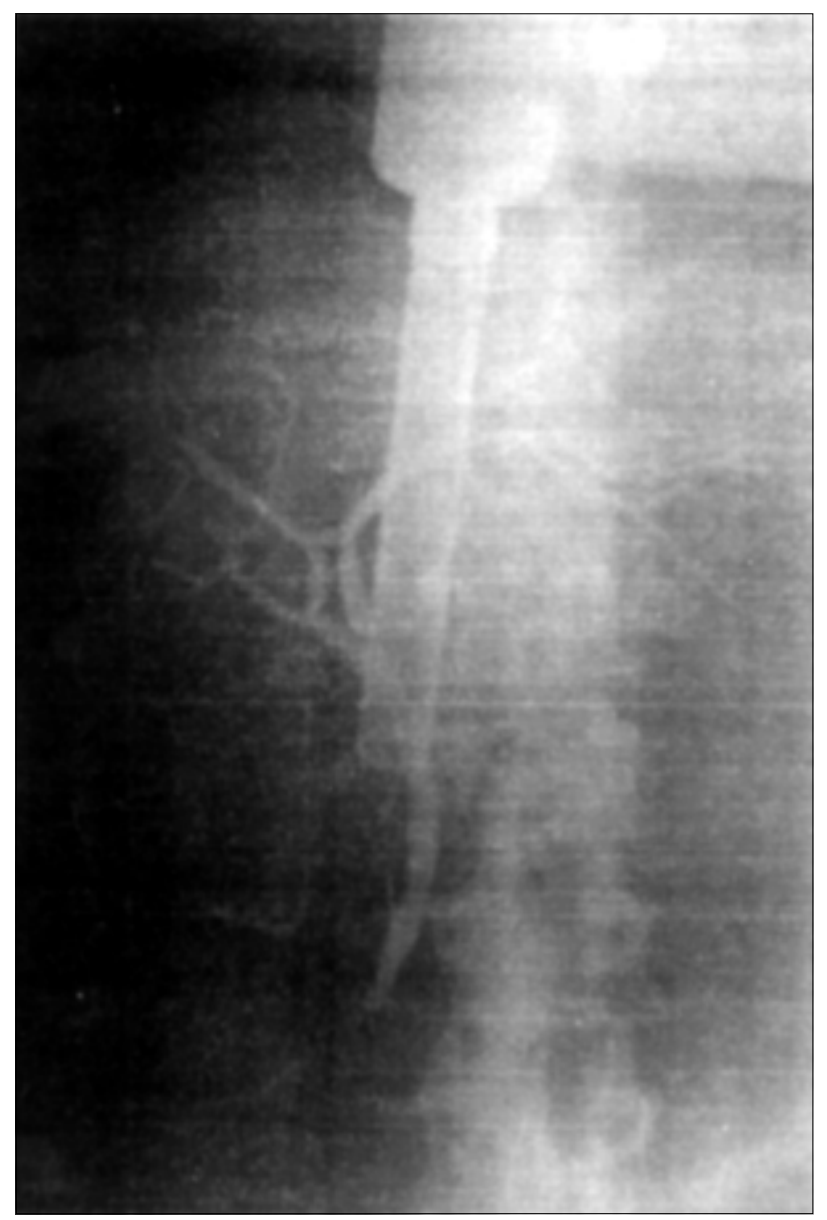

Figura 2 - Implantação do ducto cístico no hepático direito.

\section{DISCUSSÃO}

Com o advento da colecistectomia videolaparoscópica, a colangiografia peroperatória voltou a ser alvo de discussões no cenário cirúrgico nacional e internacio$\mathrm{nal}^{2,3,6,10,16,17,22-24}$.

Não existe ainda um consenso quanto à realização rotineira deste exame. Recentemente, vários autores abordaram este tema, alguns relatando a importância deste método diagnóstico, como tempo obrigatório nas colecistectomias videolaparoscópicas ${ }^{3,12,21,22}$ e outros considerando como tempo não obrigatório ${ }^{6,16-18}$.

Astudillo et al. $^{3}$ apresentaram uma casuística de 211 colecistectomias videolaparoscópicas, onde foram detectados três casos de coledocolitíase, e um paciente, com colangiografia peroperatória normal, apresentou meses depois coledocolitíase com icterícia obstrutiva. Concluíram recomendando a utilização sistemática da CPO.

Barkun et al. ${ }^{16}$ apresentaram uma casuística de 1.300 pacientes portadores de colelitíase, e 106 foram submetidos à colangiopancreatografia retrógrada endoscópica (CPRE) no período pré-operatório, tendo encontrado 50 pacientes com coledocolitíase $(3,8 \%)$. Concluíram que a

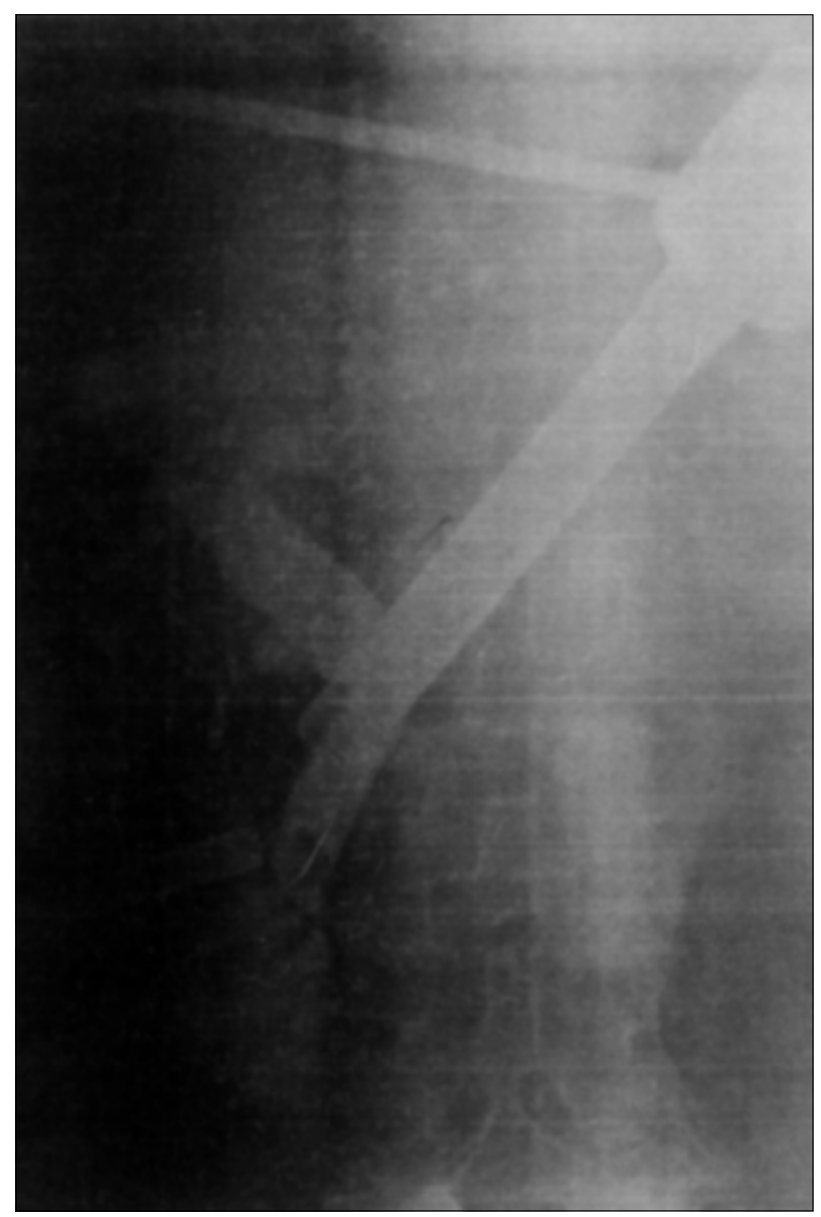

Figura 3 - Presença de cálculo no terço inferior de colédoco dilatado.

CVL pode ser realizada com segurança, sem a CPO sistemática, desde que seja realizado de rotina a CPRE no período pré-operatório.

Kuster et al. ${ }^{21}$ relataram que a colangiografia no curso de uma colecistectomia laparoscópica tem o seu valor, não só para detectar os cálculos na via biliar principal, como também para delinear a anatomia dos ductos biliares, facilitando a sua dissecção, evitando as iatrogenias e identificando as anomalias.

Larach $^{12}$, em uma casuística de 211 colecistectomias, tentou realizar a colangiografia nos últimos 59 doentes, obtendo êxito em 53. Em oito destes, foram encontradas alterações: dois casos de coledocolitíase, dois casos de cálculos encravados na união do cístico com o colédoco, duas anomalias de vias biliares e duas colangiografias falso-positivas. Concluiu que a colangiografia é um procedimento útil na prevenção das lesões das vias biliares, no diagnóstico das doenças intraductais e das anomalias das vias biliares.

Em relação aos dados demográficos, a relação entre homens e mulheres, neste estudo, foi de 3:1, compatível com os dados descritos na grande maioria da literatura pesquisada, embora já existam relatos de uma diminuição desta relação para 2:125 ou até mesmo $1: 1^{3}$. 


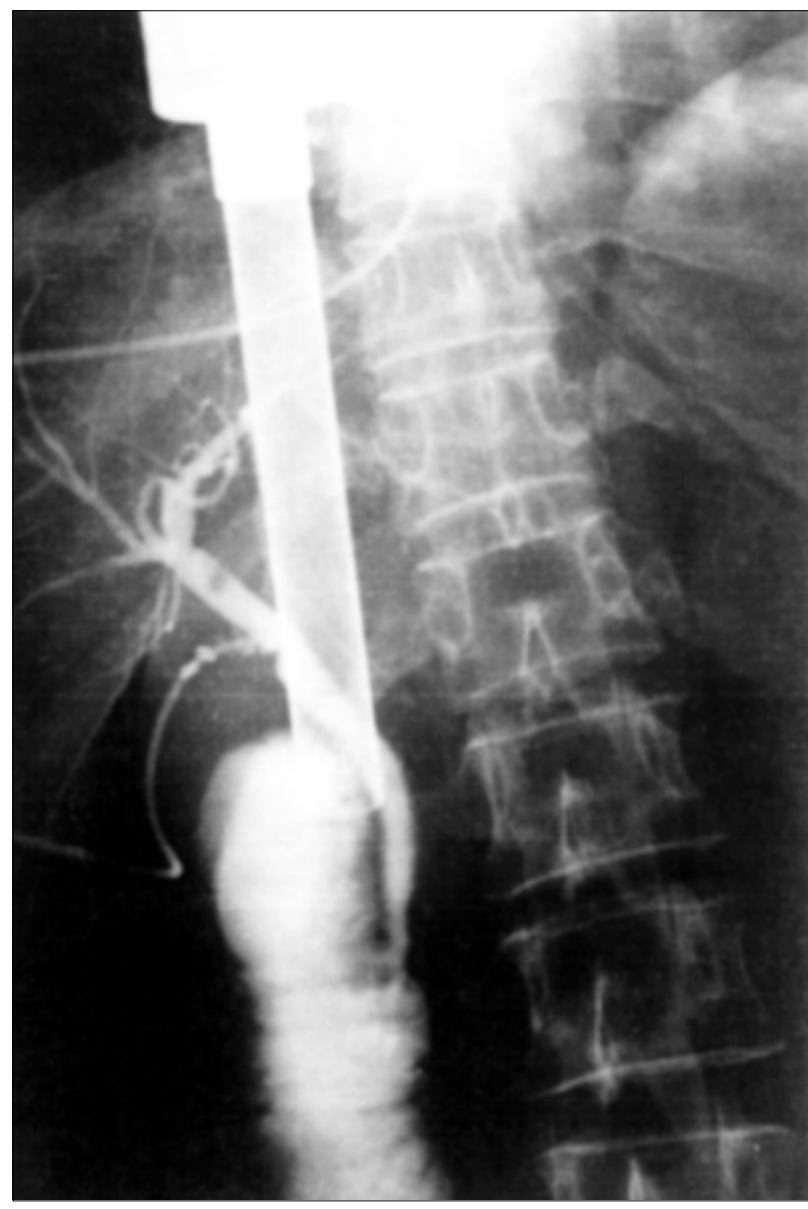

Figura 4 - Presença de cálculo no hepático comum.

A faixa etária mais acometida esteve entre 40 e 50 anos e os sinais e sintomas mais freqüentemente encontrados também estão de acordo com a maioria da literatu$\mathrm{ra}^{3,4,13,25,26}$.

A dificuldade técnica, com o conseqüente aumento do tempo operatório, consiste num dos fatores preponderantes na decisão de realizar, ou não, a colangiografia durante a colecistectomia ${ }^{3,4,13,14,20,21,22,24,27}$. Berci et al. ${ }^{2}$ demonstraram um índice de insucesso de $10 \%$, durante a tentativa de realização da CPO. D'Albuquerque et al. ${ }^{14}$ apresentaram um índice de 25\%; Astudillo et al. ${ }^{3}$ de $16 \%$; Castillo ${ }^{4}$ de $9,5 \%$; Lisboa et al..$^{13}$ de $26,5 \%$ e Fiore et al..$^{28}$ de $13,9 \%$ de insucesso. Em nossa casuística, esta percentagem atingiu $5,4 \%$.

As principais causas de insucesso na realização da CPO, como o ducto cístico fino ou fibrosado, também foram semelhantes às descritas por outros autores, com exceção da alergia ao contraste, que não foi observada nesta pesquisa. Foram relatadas também dificuldades técnicas, RX inoperante, paciente de alto risco cardiológico, aderências, dificuldades anestésicas, má posição do doente e lesão de colédoco ${ }^{3,4,13,20,21}$.

Foram realizados e valorizados, em todos os pacientes, os exames laboratoriais de bilirrubinas e aminotrans- ferases séricas, com a finalidade de encontrar algum padrão laboratorial de colestase. Outros autores utilizaram, como exame preditivo pré-operatório, a dosagem da fosfatase alcalina sérica e demonstraram um índice de significativa importância, o qual, juntamente com o tempo de doença, serviu para selecionar os pacientes com algum tipo de quadro obstrutivo extra-hepático ${ }^{13,24}$.

Outro exame pré-operatório da maior importância foi a ultra-sonografia abdominal, considerada fundamental para o diagnóstico de litíase vesicular, e também utilizada para o diagnóstico de coledocolitíase e de outras alterações das vias biliares, tais como microlitíase múltipla e dilatação das vias biliares principais ${ }^{2-4,12-14,20,22,24,26}$.

Das 237 colangiografias realizadas durante as CVL foram evidenciados seis casos de coledocolitíase e 25 casos de alterações anatômicas, como a implantação alta do ducto cístico no colédoco. Estes casos representaram 2,5\% e $10,5 \%$, respectivamente, de nossa casuística, semelhante aos índices encontrados por outros autores ${ }^{4,13}$.

Dos seis casos de coledocolitíase, em três havia a suspeita clínica, baseada nos exames pré-operatórios, enquanto nos outros três doentes não havia nenhum sintoma ou sinal clínico ou exame subsidiário pré-operatório indicativo de colestase. No primeiro, segundo e terceiro casos, os pacientes apresentavam alterações das bilirrubinas séricas, com aumento das frações direta e total, como, também, visibilização de cálculo na via biliar principal e dilatação do colédoco à ultra-sonografia do abdome superior.

Nos quarto, quinto e sexto casos, os pacientes não apresentavam sinais ou sintomas preditivos da presença de cálculos na via biliar principal, tendo sido detectados apenas durante a CPO.

Em cinco destes doentes, mesmo após a identificação peroperatória dos cálculos na via biliar principal, procedeu-se à colecistectomia isoladamente, não sendo realizada nenhuma intervenção sobre as vias biliares para a retirada destes cálculos e nem mesmo a conversão para a cirurgia convencional. Berci ${ }^{19}$ preconizou a exploração da via biliar principal e a retirada transcística dos cálculos por via laparoscópica no período peroperatório, permitindo, deste modo, a resolução em um só tempo destas eventuais intercorrências e, ainda, possibilitando submeter o paciente a um único trauma anestésico-cirúrgico.

No primeiro caso, foi decidido pela observação e acompanhamento pós-operatório do paciente, visto que a imagem da colangiografia mostrava um cálculo único e de pequeno tamanho (Figura 4), com a eventual possibilidade de o mesmo migrar para o duodeno. Até o momento, com 15 meses de evolução, o paciente apresenta-se assintomático. Esta conduta foi semelhante à adotada por Astudillo et al. ${ }^{3}$, também com bons resultados.

No segundo caso, havia sido realizada CPRE no préoperatório, com retirada de um cálculo evidenciado nesta ocasião. Devido à detecção de outros cálculos no colédoco durante a CPO (Figura 3), optou-se pela realização de uma nova retirada endoscópica dos mesmos no período pósoperatório, tendo este doente apresentado boa evolução no pós-operatório precoce e tardio. Esta conduta também foi adotada por Astudillo ${ }^{3}$. 
No terceiro, quarto e quinto casos, também foi preconizada a papiloesfincterotomia endoscópica para a retirada de cálculos, realizada com sucesso, à semelhança dos relatos de outros autores ${ }^{4,12,14}$.

No sexto caso, houve a conversão para a cirurgia convencional, objetivando a retirada de cálculos da via biliar principal. Esta decisão deveu-se a uma provável dificuldade para a retirada endoscópica posterior, visto ser o paciente portador de gastrectomia com reconstrução do trânsito à Billroth II, pois, neste caso, ocorrem duas situações difíceis para o endoscopista: o primeiro é alcançar a papila; e o segundo, a canulação dos ductos biliar e pancreático em sentido inverso ao habitualmente realizado ${ }^{30}$.

O tempo gasto para a realização da colangiografia peroperatória variou de 15 a 30 minutos (média de 20 minutos), considerado adequado também por outros autores $\mathrm{s}^{3,4,16}$.
Não foram encontrados casos de falso-positivos nesta pesquisa. No entanto, em alguns trabalhos foram descritos cerca de $2 \%$ a $16 \%$ de casos de falsa positividade ${ }^{4,26}$, possivelmente devido à presença de ar nos ductos, pela má qualidade das radiografias ou pela técnica imperfeita da colangiografia ${ }^{21,24,26}$.

Nesta casuística, não tivemos casos de lesão iatrogênica das vias biliares ou de complicações maiores no período pós-operatório, ao contrário dos relatos de alguns autores, em que os índices de lesões da via biliar principal e de complicações graves (como coleperitônio ou hemorragias), variam de $1,5 \%$ a $2 \%$ dos casos $4,11,18,26$.

A colangiografia peroperatória, realizada sistematicamente mostrou ser um procedimento seguro durante a colecistectomia videolaparoscópica, com a capacidade de identificar as alterações anatômicas existentes e de demonstrar a eventual presença de cálculos na via biliar principal, reduzindo ainda a possibilidade de lesões iatrogênicas.

\begin{abstract}
Background: We present our results from peroperative cholangiography (POC) systematically performed during video laparoscopic cholecystectomies (VLC). Method: From January 1998 to December 1999, VLP was performed in 250 non-selected patients from both sexes, being 65 males (26\%) and 185 females (74\%), independent of the presence or not of clinical signals, laboratorial and ultrasonographics results or of other diagnostic method, indicatives of choledocholithiasis or other biliary ducts pathologies Results: POC could be performed in 94,6\% of the cases. It was found 31 modifications (13\%), being the most important six cases of choledocholithiasis (2,5\%) and 25 cases of anatomical anomalies (10,5\%). Conclusions: POC during VLC showed to be a safe procedure, with no complications, defining anatomy, forewarning biliary injuries and allowing assymptomatic choledocholithiasis detection.
\end{abstract}

Key words: Cholangiography; Cholecystectomy; Vesicular lithiasis; Video laparoscopy

\title{
REFERÊNCIAS
}

1. Mirizzi PL. Operative cholangiography. Surg. Gynecol. Obstet., 1937, 65: 702-710.

2. Berci G, Sackier JM, Paz-Partlow M. Routine or selected intraoperative cholangiography during laparoscopic cholecystectomy? Am J. Surg., 1991, 161 (3): 355360.

3. Astudillo PS, Minuzzi F, Allende FS et al. Valor de la colangiografia operatoria em la cirurgia biliar laparoscopica. Rev. Argent. Cirug., 199466 (1-2): 19-25.

4. Castillo JLT. Colangiografia laparoscopica. Rev. Chil. Cir., 1995, 47 (6): 599-604.

5. Hirono Y, Takita $\mathrm{Y}, \mathrm{Nitta} \mathrm{N}$ et al. Double cystic duct found by intraoperative cholangiography in laparoscopic cholecystectomy. Surg. Laparosc. Endosc., 1997, 7(3): 263-265.

6. Chaib E, Freire ANM, Cantanhede AVR et al. É necessário realizar colangiografia operatória como rotina nas colecistectomias? Arq. Gastroenterol., 1990, 27 (1): $10-13$.
7. Grace PA, Qureshi A, Burke P et al. Selective cholangiography in laparoscopic cholecystectomy. Br. J. Surg, 1993, 80 (2): 244-246.

8. Bravo Leon E. Enfrentamiento quirúrgico de la litiasis biliar. Rev. Chil. Cir., 1994, 46 (6): 604-615.

9. Silverstein JC, Wavak E, Millikan KW. A prospective experience with selective cholangiography. Am. Surgeon, 1998, 46 (6): 654-659.

10. Fontes PRO, Nectoux M, Eilers RJ et al. Colangiografia transoperatória em colecistectomia laparoscópica. Rev. Col. Bras. Cir., 1998, 25 (11-12):383387.

11. Ladocsi LT, Benitez LD, Filippone DR et al. Intraoperative cholangiography in laparoscopic cholecystectomy: A Review of 734 consecutive cases. Am. Surgeon, 1997, 63 (2): 150-156.

12. Larach JS. Colangiografia laparoscopica: tecnica, indicaciones, resultados. Rev. Chil. Cir., 1992, 44 (2): 192198. 
13. Lisboa FF, Melo Jr. FA, Dantas SJL et al. Colangiografia intra-operatória na colecistectomia videolaparoscópica: análise de 268 casos. Rev. Col. Bras. Cir., 1995, 22 (4): 217-222.

14. D’Albuquerque LAC, Miranda MP, Genzini T et al. Colecistectomia laparoscópica: análise de 40 casos. Anais Paul. Med. Cir, 1992, 119 (2): 16-28.

15. Iribarren $\mathrm{C}$, Colombo MD, Ortiz JA et al. La colangiografia operatoria en el paciente sin signos de litíasis coledociana. Rev. Argent. Cirurg., 1992, 63 (1-2): 37-46.

16. Barkun JS, Fried GM, Barkun AN et al. Cholecystectomy without operative cholangiography: implications for common bile duct injury and retained common bile duct stones. Ann. Surg, 1993, 218 (3): 371-379.

17. Schröder TM, Linko KY. Laparoscopic cholecystectomy without cholangiography: a study of 151 consecutive cases. Eur. J. Surg, 1995., 161: 421-424.

18. Gadacz RT. U.S. Experience with laparoscopic cholecystectomy. Am. J. Surg, 1993., 165 (4): 450-454.

19. Berci G. Biliary ductal anatomy and anomalies: the role of intraoperative cholangiography during laparoscopic cholecystectomy. Surg. Clin. North Amer., 1992, 72 (5): 1069-1075.

20. Gama-Rodrigues J, Bresciani C. A Colecistectomia por videolaparoscopia. In: Mincis M. Gastroenterologia \& Hepatologia: Diagnóstico e Tratamento. São Paulo, Lemos Editorial, 1997. pp. 815-825.

21. Kuster GGR, Gilroy S, Graefen M. Intraoperative cholangiography for laparoscopic cholecystectomy. Surg. Gynecol. Obstet, 1993, 176 (5): 411-417.

22. Pekolj J, Santibanes E, Sívori JA et al. Tratamiento de la litíasis coledociana por via transcística durante la colecistectomia laparoscópica. Rev. Argent. Cirurg, 1995, 69 (1/2): 10-17.

23. Galloway SW, Blazeby JM, Tulloh BR et al. Selective cholangiography with laparoscopic cholecystectomy: review of a protocol. Eur. J. Surg, 1996, 162: 373-378.

24. Morino M. Colangiografias intraoperacionais. In: Meinero M, Melotti G, Mouret PH. Cirurgia laparoscópica. Buenos Aires, Argentina, Editorial Médica Panamericana. 1996, pp. 166-169.
25. Dani R, Portella FW, Nogueira CED. Litíase biliar. In: Dani R, Castro LP. Gastroenterologia Clínica. 3ed.v.1. Rio de Janeiro, Guanabara Koogan, 1993. pp. 1516-1540.

26. Benson EA, Wegwood KR, Axon ATR et al. Coledocolitíase. In: Coelho J. Aparelho Digestivo: Clínica e Cirurgia. 2ed.v.1. Rio de Janeiro, Medsi, 1996. pp. 13211334.

27. Perin CA. Colecistocolangiografia intra-operatória na colecistectomia laparoscópica. Rev. Col. Bras. Cir, 1995., 22 (4): 195-200.

28. Fiore NF, Ledniczky G, Wiebke EA et al. An analysis of perioperative cholangiography in one thousand laparoscopic cholecystectomies. Surgery, 1997, 122 (3): 817-823.

29. Franceschi D, Brandt C, Margolin D et al. The management of common bile duct stones in patientes undergoing laparoscopic cholecystectomy. Am. Surgeon, 1993 , 59 (8); 525-532.

30. Sobreira RS, Patrício CE. Colangiografia endoscópica retrógrada. In. Sociedade Brasileira de Endoscopia Digestiva. Endoscopia Digestiva. Endoscopia Digestiva. 3ed. Rio de Janeiro. Medsi, 2000. pp. 21-26.

Endereço para correspondência:

Dr. Edson Yuzur Yasojima

Hospital Amazônia

Trav. Nove de Janeiro, 1.267

66060-370 — Belém-PA 